\title{
BMJ Open Individual participant data systematic reviews with meta-analyses of psychotherapies for borderline personality disorder
}

Ole Jakob Storebø (D) ,1,2 Johanne Pereira Ribeiro (D) ,', Mickey T Kongerslev, ${ }^{3}$ Jutta Stoffers-Winterling (D) , ${ }^{4}$ Mie Sedoc Jørgensen, ${ }^{1}$ Klaus Lieb, ${ }^{5}$ Anthony Bateman, ${ }^{6,7}$ Richard Kirubakaran, ${ }^{8}$ Nicolas Dérian, ${ }^{9}$ Eirini Karyotaki (D) , ${ }^{10}$ Pim Cuijpers (D),${ }^{10}$ Erik Simonsen ${ }^{1}$

To cite: Storebø 0J, Ribeiro JP, Kongerslev MT, et al. Individual participant data systematic reviews with meta-analyses of psychotherapies for borderline personality disorder. BMJ Open 2021;11:e047416. doi:10.1136/ bmjopen-2020-047416

- Prepublication history and additional supplemental material for this paper are available online. To view these files, please visit the journal online. To view these files, please visit the journal online (http://dx.doi. org/10.1136/bmjopen-2020047416).

Received 30 November 2020 Accepted 04 June 2021

Check for updates

(C) Author(s) (or their employer(s)) 2021. Re-use permitted under CC BY-NC. No commercial re-use. See rights and permissions. Published by BMJ.

For numbered affiliations see end of article.

Correspondence to Professor Ole Jakob Storebø; ojst@regionsjaelland.dk

\section{ABSTRACT}

Introduction The heterogeneity in people with borderline personality disorder (BPD) and the range of specialised psychotherapies means that people with certain BPD characteristics might benefit more or less from different types of psychotherapy. Identifying moderating characteristics of individuals is a key to refine and tailor standard treatments so they match the specificities of the individual participant. The objective of this is to improve the quality of care and the individual outcomes. We will do so by performing three systematic reviews with metaanalyses of individual participant data (IPD). The aim of these reviews is to investigate potential predictors and moderating patient characteristics on treatment outcomes for patients with BPD.

Methods and analysis We performed comprehensive searches in 22 databases and trial registries up to 0ctober 6th 2020. These will be updated with a top-up search up until June 2021. Our primary meta-analytic method will be the one-stage random-effects approach. To identify predictors, we will use the one-stage model that accounts for interaction between covariates and treatment allocation. Heterogeneity in case-mix will be assessed with a membership model based on a multinomial logistic regression where study membership is the outcome. $A$ random-effects meta-analysis is chosen to account for expected levels of heterogeneity.

Ethics and dissemination The statistical analyses will be conducted on anonymised data that have already been approved by the respective ethical committees that originally assessed the included trials. The three IPD reviews will be published in high-impact factor journals and their results will be presented at international conferences and national seminars.

PROSPERO registration number CRD42021210688.

\section{INTRODUCTION}

Borderline personality disorder: diagnosis and treatment

The polythetic approach to diagnosing borderline personality disorder (BPD), gives 256 ways of meeting the criteria for a BPD diagnosis. ${ }^{1}$ This means that apart from
Strengths and limitations of this study

- These individual participant data (IPD) reviews are the first to systematically review and investigate psychotherapy for people with borderline personality disorder using IPD.

- The IPD reviews will provide information on moderators and predictors in patients with borderline personality disorder that predict who may benefit most from which type of specialised psychotherapy.

- IPD allow for a more precise risk of bias assessment and decreases the amount of unclear risk of bias in many of the included trials.

- A limitation to IPD reviews in general is that data retrieval can be challenging.

- The IPD reviews are limited to the outcomes and patient characteristics that have been assessed in the included trials.

meeting the general diagnostic criteria for personality disorder $(\mathrm{PD})$, the patients also need to fulfil five or more of the nine specific BPD criteria according to the current Diagnostic and Statistical Manual of Mental Disorders (DSM) classification system. ${ }^{1}$ This makes the population with BPD highly heterogeneous. This fact is exacerbated by the common co-occurrence of many other psychiatric and somatic conditions. Co-occurring psychiatric conditions, for example, life-threatening eating disorders or substance use dependence, are often persistent and may impede BPD treatment. ${ }^{2-4}$ People with BPD need effective treatment due to the considerable psychological suffering of those concerned, ${ }^{5}$ the high burden experienced by their families and significant others, ${ }^{67}$ the significant impact they have on mental health services given their frequent use, ${ }^{89}$ as well as 
the association with sustained functional impairment, ${ }^{10}$ physical illness ${ }^{11}$ and premature death. ${ }^{12-14}$

The prevalence of BPD in the general population is estimated to be $1.8 \%,{ }^{15}$ and the diagnosis is one of the most common in the psychiatric system. ${ }^{16}$ In addition to the effects on the individuals and their relatives, the annual direct total costs for the Danish health sector is roughly $€ 53000$ per patient with BPD per year. This number is 16 times the costs of general population controls according to a recent nationwide study by Hastrup and colleagues. ${ }^{17}$ From an economic perspective alone, this calls for more effective treatments for people with BPD and a precisely targeted use of resources.

Most people with BPD will receive psychological interventions because drugs are not effective for BPD core symptoms. ${ }^{4} 18$ Psychological interventions are often provided for relatively long periods (e.g., 1 year or longer).$^{20}{ }^{21}$ Psychotherapy is thus the current treatment of choice for patients with BPD. ${ }^{22}$ Most people in treatment for BPD receive long-term psychotherapeutic intervention, ${ }^{421}$ however not all individuals in need have access to adequate treatment, even in highly developed countries. ${ }^{23}$ A recent review of European guidelines on diagnosing and treating PDs reported, that psychotherapy was the first-line treatment recommended in all countries. ${ }^{22}$

A broad range of specialised psychotherapies for BPD is available. ${ }^{24-26}$ These therapies are usually precisely structured and manualised ${ }^{24}$ and are delivered in individual therapy format or as combined individual- and grouptreatment. Most BPD-specific psychological interventions involve multimodal therapy, treatment contracts, actively taking measures to minimise premature noncompletion of treatment, providing a protocol for crisis intervention and stimulating the participant's sense of agency. ${ }^{24} 25$ 27-30 Psychotherapeutic treatments for BPD are based on a variety of different therapeutic schools, for example, psychodynamic, cognitive-behavioural or client-centered/humanistic therapy. ${ }^{31}$ However, there has been a development of multiple psychotherapeutic treatments that are more disorder-specific (i.e., specifically adapted for BPD) within the last three decades. This development is due to the disorder-inherent challenges that individuals diagnosed with BPD often face and pose in treatment. Among the specific psychological interventions for people diagnosed with BPD, the most commonly researched and used ones are: dialectical behaviour therapy, ${ }^{32} 33$ mentalisation-based treatment, ${ }^{34-36}$ systems training for emotional predictability and problem solving, ${ }^{37}$ transference-focused therapy, ${ }^{38}{ }^{39}$ cognitive analytic therapy ${ }^{404}$ and schema-focused therapy. ${ }^{42} 43$ The treatment of BPD is very complex due to the complexity of the pathology itself, but tailored treatments can improve the outcomes. Therefore, we want to systematise the use of treatments to match patient characteristics by conducting these individual participant data (IPD) reviews.

Storeb $\varnothing$ and colleagues ${ }^{26}$ published a Cochrane review in May 2020 that investigated the beneficial and harmful effects of psychotherapeutic treatments for people with
BPD. Their findings showed, that all major types of psychotherapies for BPD had a modest positive average effect at group level. It is likely however that the participants' individual responses differed in relation to certain self-inherent characteristics. Therefore, data are now needed at the level of the individual patient to find out for whom the different specialised psychotherapies may have a greater or smaller effect (i.e., what type of psychotherapy will have the largest treatment effect, when taking the personal and clinical characteristics of the participant into consideration).

Given the heterogeneity of individuals affected by BPD and the availability of several effective treatments of different theoretical orientations ${ }^{26}$ it is possible that individuals with certain characteristics might benefit to a higher extent from some treatments, and less from others.

Identifying such patient characteristics may allow for a more refined and individualised treatment, and optimise the treatment quality and effect for patients with BPD. ${ }^{44}$ Research identifying BPD characteristics that affect the outcome of the various treatments is therefore needed.

As called for by Barber and Solomonov, ${ }^{45}$ we attempt to find and match the most effective specialised psychotherapeutic treatments with the needs of the individual patient, based on personal and clinical characteristics. This way we are effectively moving towards a personalised approach to psychotherapeutic treatment.

\section{Purpose of the individual participants data reviews}

The preceding Cochrane review of psychological therapies for $\mathrm{BPD}^{26}$ provided an initial overview of the research in the area, and presented results based on analyses of aggregated data. This Cochrane review can be considered a first step in the research process. This project is the next step which focuses on predictors and moderators of outcomes. ${ }^{46}$

We define predictors as a collection of parameters (demographic, clinical, or biologic) that influence the likelihood of specific outcomes to occur.

Moderators are special cases of predictors defined as baseline parameters (demographic, clinical, or biologic) affecting the likelihood of a specific event to occur in one situation compared with another. ${ }^{47}$

This project aims to provide tangible advice for practitioners and people affected by the disorder on how to select the psychotherapeutic treatment deemed to have the most effective outcome when considering patient characteristics. Overall, this project attempts to ensure an evidence base that might contribute to more people with BPD receiving a treatment that is adapted to the individual's needs. To investigate these characteristics, we will perform three systematic reviews with meta-analyses of psychotherapies for BPD using IPD. IPD meta-analyses are particularly well suited for this project because all the raw data from the included trials are used, which allows for a detailed exploration of causes of heterogeneity. ${ }^{48}$ IPD reviews are closely related to personalised medicine 
where it is important to understand for whom, and under what conditions, treatment exerts the best effect. Furthermore, findings of these reviews are likely to inform future treatment guidelines.

\section{IPD review methodology}

Although the IPD methodology is still rather new, IPD reviews have generally had a substantial impact on clinical practice and research. ${ }^{46}$

When IPD for each participant in clinical trials are available, these can be used to individualise the results of clinical trials. ${ }^{24}$ There are already several examples of recent IPD reviews that have decreased the knowledge-gap in somatic research areas. ${ }^{49-51}$ Within the psychiatric field, IPD reviews have been used to investigate treatment effects across various patient groups, with direct implications for clinical practice ${ }^{52-57}$ When conducting extensive searches in relevant databases however, we found no IPD review that investigated psychotherapy for BPD.

The use of IPD can promote standardisation of data in analyses and allows for direct extraction of data to outcomes, independently of how these were reported in the original trial publication. Studies that use IPD show a greater power in detecting effect differences in outcomes between individuals. ${ }^{58}$ This can provide valuable information about responders and non-responders to the different types of treatments. Analyses based on IPD data also allow for the use of more sophisticated statistical methods. ${ }^{59}$ In particular, IPD may allow for exploring causes of heterogeneity such as baseline differences, selection criteria, dose and duration of treatments received by participants in control groups, and differential negative effects of the treatments. Missing data can also be handled in a more standardised manner in IPD reviews. Furthermore, access to IPD data allows for a more reliable risk of bias assessment due to better insight into the original data. Finally, IPD allows us to perform subgroup analyses that have not previously been conducted, thereby answering new and pressing research questions concerning how to optimise treatments for BPD for the individual participant. ${ }^{48}$

\section{Objectives}

This protocol describes three planned IPD reviews, each aiming to answer different salient research questions. These are research questions that remain pertinent based on the prior literature, and especially the recently published Cochrane review on the topic ${ }^{26}$.

IPD review 1: BPD symptom severity and interpersonal functioning (1.1) What are the effects of different psychotherapies when compared with unspecific controls (e.g., treatment usual (TAU), wait-list (WL) or no-intervention (NI) and specific psychotherapeutic interventions for people with BPD on the primary outcomes: BPD symptom severity and interpersonal functioning?

(1.2) What are the moderators of the differential efficacy between psychotherapy versus control conditions in reducing BPD symptom severity and increasing interpersonal functioning?

(1.3) What are the prognostic factors and effect moderators associated with the secondary outcomes: serious and non-serious adverse events?

\section{IPD review 2: quality of life and psychosocial functioning}

(2.1) What are the effects of different psychotherapies when compared with unspecific controls (e.g., TAU, WL or NI) and specific psychotherapeutic interventions for people with BPD on the primary outcomes: quality of life and psychosocial functioning?

(2.2) What are the moderators of the differential efficacy between psychotherapy versus controls in quality of life and psychosocial functioning?

(2.3) What are the prognostic factors and effect moderators associated with the secondary outcomes: serious and non-serious adverse events?

\section{IPD review 3: self-harm and suicide-related outcomes}

(3.1) What are the effects of different psychotherapies when compared with unspecific controls (e.g., TAU, WL or NI) and specific psychotherapeutic interventions for people with BPD on the primary outcomes: self-harm and suicide-related outcomes?

(3.2) What are the moderators of the differential efficacy between psychotherapy versus controls in reducing self-harm and suicide-related outcomes?

(3.3) What are the prognostic factors and effect moderators associated with the secondary outcomes: serious and non-serious adverse events?

\section{METHOD AND ANALYSIS \\ General approach}

This protocol follows the general guidance provided as part of the Preferred Reporting Items for Systematic Reviews and Meta-Analyses-IPD statement ${ }^{60}$ (see checklist $\mathrm{S} 1$ in the online supplemental material).

\section{Search criteria}

To meet our inclusion criteria, at least $70 \%$ of participants in a trial are required to have a formal diagnosis of BPD according to the Diagnostic and Statistical Manual of Mental Disorders, third edition, revised (DSM-III-R) and onwards. ${ }^{1}$ We will include trials with subsamples of people with BPD when data are provided separately on BPD participants. We will not include trials that focus on people with mental impairment, organic brain disorder, dementia or other severe neurologic/neurodevelopmental diseases or people with medical health issues, for example, cancer or HIV.

The search will not be limited by language, year of publication, or type of publication. We will seek translation of relevant sections of articles that are not in English.

\section{Search method for identification of studies}

Our search strategy for eligible studies will be based on the searches conducted in the prior Cochrane review on 
psychological therapies for BPD. ${ }^{26}$ These searches will be updated with a top-up search which is described in detail below (see the online supplemental material S2 for search string).

\section{Databases}

We will search for eligible studies in the following 22 databases and registries: Cochrane Central Register of Controlled Trials, MEDLINE Ovid, Embase Ovid, CINAHL EBSCOhost, PsycINFO Ovid, ERIC EBSCOhost, BIOSIS Previews, Web of Science Core Collection Clarivate Analytics, Sociological Abstracts ProQuest, LILACS, OpenGrey, JISC Library Hub Discover (previously COPAC), Proquest Dissertations and Theses Global, DART Europe E-Theses Portal, Networked Digital Library of Theses and Dissertations, Australian New Zealand Clinical Trials Registry, Clinicaltrials.gov, EU Clinical Trials Register, Open Trials, ISRCTN Registry, Be Part of Research, WHO International Clinical Trials Registry Platform.

\section{Types of studies}

The studies that will be included in our search are randomised clinical trials (RCTs) that compare psychotherapeutic treatments for BPD with unspecific controls (eg, TAU, WL, and NI) and specific psychotherapeutic treatments.

\section{Population}

The studies will include people of all ages, any gender, in any setting, with a formal, categorical diagnosis of BPD according to the DSM, third edition (DSM-III; APA 1980), DSM-III-R (APA 1987), DSM, fourth edition (DSMIV; APA 1994), DSM, fourth edition, text revision (DSMIV-TR; APA 2000) and DSM, fifth edition (DSM-5; APA 2013) or the emotionally unstable PD, borderline type in International Classification of Diseases and Related Health Problems, 10th version (WHO 1993), with or without comorbid conditions. ${ }^{26}$

\section{Intervention}

We will search for well-defined theory-driven psychological interventions regardless of theoretical orientation (e.g., psychodynamic therapy, cognitive behavioural therapy, systemic therapy, or eclectic therapies designed for BPD treatment), in any kind of treatment setting (e.g., inpatient, outpatient, or day clinic) and mode (individual, group or combined therapy).

\section{Study selection}

The paper titles and abstracts identified in the top-up search will be independently screened by two members of the project group to remove those that are clearly ineligible. Similarly, two reviewers will read the full-text articles independently. Disagreements about study inclusion will be resolved by discussion with a third review author. All trials excluded from the review after the full-text level will be given reasons for exclusion.

\section{Quality assessment}

Study quality will be assessed by two reviewers from the project group who will independently evaluate the studies using the updated Cochrane Risk of Bias tool in the quality assessment of included studies. ${ }^{61}$

Studies will be rated on each criterion with either 'low risk', 'high risk' or 'some concerns'. Each study as a whole will be rated according to its highest risk of bias in any of the assessed domains, that is, if any domain is judged as having a high risk of bias, the whole study will be classified as 'high risk of bias'. We will assess the following domains: (1) bias arising from the randomisation process, (2) bias due to deviations from the intended interventions, (3) bias due to missing outcome data, (4) bias due to measurement of the outcome and (5) bias due to selective reporting. ${ }^{59}$

\section{Data collection process}

To be able to get raw data from the included RCTs, we will obtain contact information through the included publications or by an online search. We will contact the authors of each included RCT and provide them with the IPD review protocol and a cover letter explaining what the study is about. If we receive no response, we will send a reminder after 1 week and again after 1 month before excluding the trial for unavailability.

\section{IPD-BPD consortium}

All RCT authors will be invited to be part of an IPD-BPD consortium that the project group will establish. The name of this consortium will be 'IPD-BPD'. The aim of this task force is to support the project, make it easier to have authors participate, to increase awareness within the public and clinical community, and to help with dissemination of results. All RCT authors will be invited to be co-authors of the IPD reviews.

\section{Developing the IPD-BPD database}

IPD will be extracted from all included RCTs where the authors are willing to share their data. The IPD will be exported and integrated into a spreadsheet. A template spreadsheet will be created and pilot-tested. We will need data from all randomised patients (intention-to-treat samples) of all included trials. We will make a list of variables that we need and send this to the authors of the included trials. Furthermore, we will ask for the formal data codes and time points at which data were collected.

Raw data (de-identified data) can be transferred by a secure electronic transfer. The data sent from authors will be checked for completeness and accuracy. We will compare the participant numbers, descriptive data, and outcome data to the reported data in the original peerreviewed article. If any irregularity is present, the issue will be discussed with the study authors for clarification. Raw datasets will be saved in their original formats and then exported into a common format. The data will be stored on a secure server. We will rename the variables for each study in a consistent manner. All individual datasets 
will be merged into one large IPD dataset that takes the study clusters into account. ${ }^{62}$

\section{Data items}

\section{Primary outcomes}

The same primary outcomes that were used in the 2020 Cochrane review of aggregated data ${ }^{26}$ will be used in the three IPD reviews: BPD severity, self-harm, suicide-related outcomes and psychosocial functioning. These will be complemented by two more outcomes, that is, quality of life and interpersonal functioning. Quality of life takes the perspective of individuals affected and provides a very direct measure of treatment effects from their stance. Interpersonal functioning was included along with quality of life as it reflects one of the core problems of BPD (besides impulsivitydysregulative- and emotionally dysregulative pathology), and is very likely to affect the individual's well-being and psychosocial and vocational functioning.

Primary outcomes will be measured by the use of standardised psychometric rating scales. We will include both self-rated and observer-rated measures.

\section{IPD review 1}

(1.1) BPD symptom severity, for example, assessed by the Zanarini Rating Scale for Borderline Personality Disorder (Zan-BPD),${ }^{63}$ the Borderline Personality Disorder Severity Index, fourth version (BPDSI-IV) ${ }^{64}$ or the Clinical Global Impression Scale for people with Borderline Personality Disorder (CGI-BPD). ${ }^{65}$

(1.2) Interpersonal functioning, for example, assessed bythe Inventory of Interpersonal Problems ${ }^{66}$, or the relevant item or subscale on the Zan-BPD, ${ }^{63}$ CGI-BPD, ${ }^{65}$ BPDSI-IV. ${ }^{64}$

\section{IPD review 2}

(2.1) Quality of life, for example, assessed by the The Quality of Life Satisfaction and Enjoyment ${ }^{67}$ or the EuroQol five-dimensional. ${ }^{68}$

(2.2) Psychosocial functioning, for example, assessed by the Global Assessment Scale, ${ }^{69}$ the Global Assessment of Functioning Scale ${ }^{70}$ or the Social Functioning Questionnaire. $^{71}$

\section{IPD review 3}

(3.1) Self-harm, in terms of the proportion of participants with self-harming behaviour, or assessed by for example, the Deliberate Self-harm Inventory ${ }^{72}$ or the Self-harm Behaviour Questionnaire. ${ }^{73}$

(3.2) Suicide-related outcomes, for example, assessed by the Suicidal Behaviours Questionnaire, ${ }^{74}$ or the Beck Scale for Suicidal Ideation, ${ }^{75}$ or in terms of the proportion of participants with suicidal acts.

\section{Secondary outcomes}

Adverse effects will be measured by the use of standardised psychometric rating scales, such as the Systematic Assessment for Treatment Emergent Events, ${ }^{76}$ by laboratory values or spontaneous reporting. We will divide the reported adverse effects into severe and non-severe, according to the International Committee of Harmonization guidelines. ${ }^{77}$ We will define serious adverse effects as any event that led to death, was life-threatening, required inpatient hospitalisation or prolongation of existing hospitalisation, resulted in persistent or significant disability, or any important medical event that may have jeopardised the participant's health or required intervention to prevent one of the aforementioned outcomes occurring. We will consider all other adverse effects to be non-serious. Additionally, deterioration will be examined.

\section{Effect predictors and moderators}

We want to know which participant characteristics predict an improvement of the primary outcomes for the three IPD reviews regardless of treatment allocation (predictor variables): IPD review 1: BPD symptom severity and interpersonal functioning; IPD review 2: Quality of life and psychosocial functioning and IPD review 3: Self-harm and suicide-related outcomes. (see figure 1).

We also intend to identify moderators, that is, variables that affect outcomes based on treatment allocation. ${ }^{78}$ Moderators differentiate between the effects of two treatments, and predictors refer to prognostic factors. ${ }^{78}$

Participant characteristics will be included in the analyses, if they are consistently reported, available across datasets and justify inclusion based on prior literature that identifies them as potential predictors or moderators. ${ }^{78}$ To minimise the risk of multiplicitiy, i.e. falsely rejecting the null hypothesis, we will include only the six to eight most important moderators. We will then adjust the $\mathrm{P}$ values and CIs of the primary and secondary outcomes for multiplicity using the method described by Jakobsen et $a .^{79}$ Such characteristics could be age at baseline, sex, ethnicity, country of birth, education status, employment status, marital status, severity of BPD, psychosocial impairment, treatment adherence, comorbidity, previous mental illness, medications (psychotropic), mental illness in the family, socioeconomic factors, criminal behaviour, personality traits, previous trauma, IQ, suicide attempts, anger, chronic feelings of emptiness, impulsivity, interpersonal problems, abandonment, psychotic-like symptoms, depression, and self-harm incidents. We will examine the published papers and verify which moderators are investigated. We will include all moderators that are investigated in at least two studies.

\section{Data analysis}

Our primary meta-analytic method will be the one-stage random-effects approach, which is particularly suitable for investigating predictors and moderators compared with the two-stage method. The one-stage random-effects method is also less influenced by the expected small size of some the studies included in the planned meta-analyses. ${ }^{80}$

To identify predictors, we will use the one-stage model that accounts for interaction between covariates and treatment allocation. Covariates with statistical evidence for association with the outcome will be added in a unique 


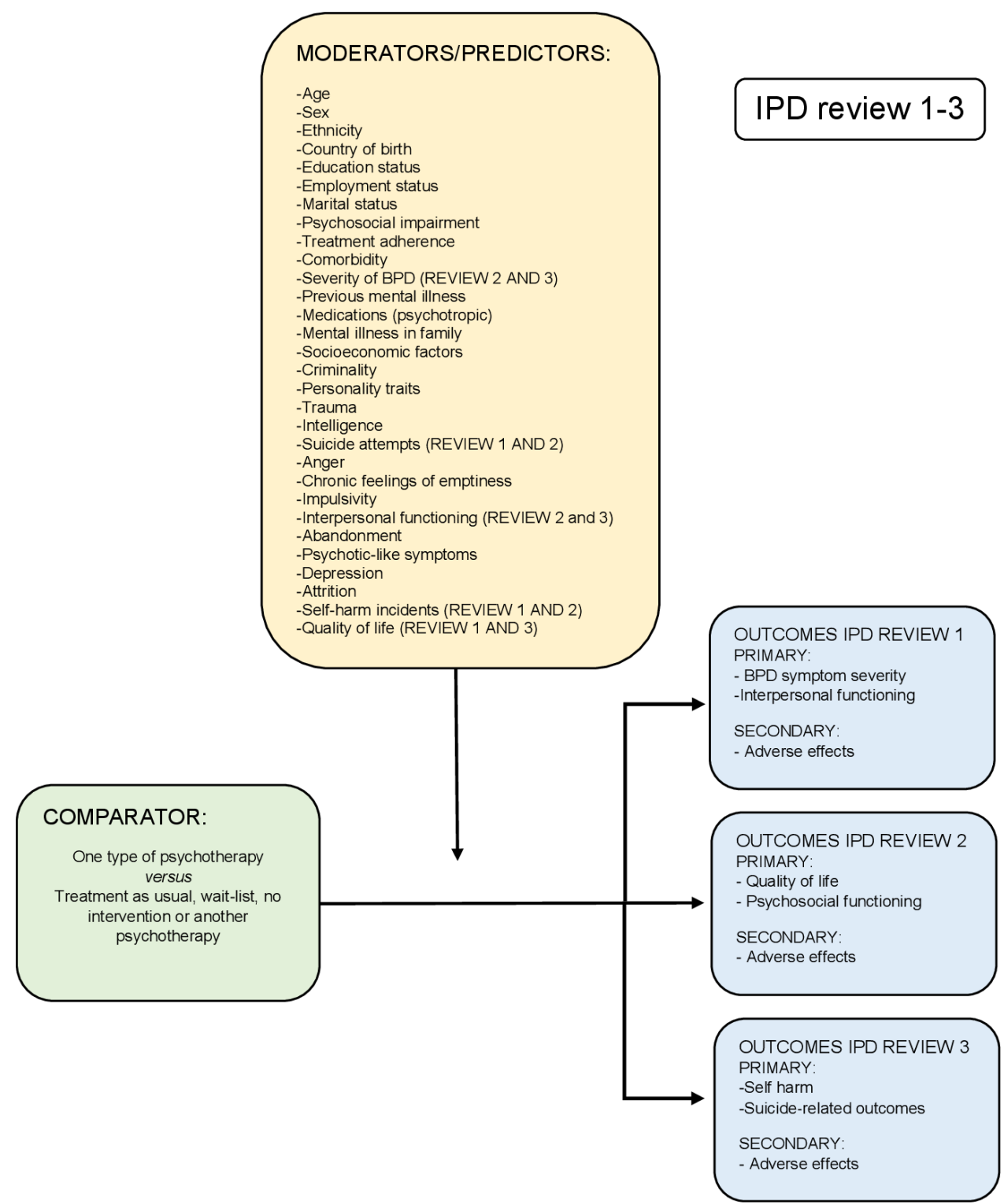

Figure 1 Comparisons, moderators and outcomes in IPD reviews 1-3. BPD, borderline personality disorder; IPD, individual participant data.

global model. Significant association $(\mathrm{p}<0.05)$ with the outcome in the global model will then be used to identify the predictors. Similarly, we will use a one-stage approach to identify moderators by investigating the interaction between selected covariates and the treatments, one covariate at the time. ${ }^{78}$ To account for potential ecological bias, covariates will be transformed at study-level before analysis using the proper methodology. ${ }^{81}$

Datasets will be checked for their completeness and integrity. To handle missing values, we will use multiple imputation under the missing at random assumption. ${ }^{82}$ Missing data will be imputed within each original study before data of the individual studies are pooled. A sensitivity analysis will be conducted using a pattern-mixture approach. ${ }^{83}$

Heterogeneity in case-mix will be assessed using a membership model based on a multinomial logistic regression where study membership is the outcome.
The derived c-statistics will reflect the difference in baseline characteristics and outcome. ${ }^{84}$ As a certain level of heterogeneity is expected (e.g., due to differences in study populations, types of psychotherapy, or differences in the control group) a random-effects meta-analysis is chosen to account for these variations.

All analyses will be conducted using a well-established statistical platform providing ready-to-use packages and libraries to perform such analyses, like STATA. ${ }^{85}$

\section{Subgroup analyses}

In addition, we will perform meta-analyses including only studies classified as 'low risk' of bias to assess the impact of studies of lower methodological quality and type of control conditions on the findings. When possible, a similar approach will be used to compare studies based on differences in the criteria for the risk of bias. ${ }^{86}$ 
Difference between included and not-included studies in the IPD review

We will compare the dataset on the primary outcomes from the previously published Cochrane review ${ }^{26}$ with the data included in the present IPD reviews. If there are any discrepancies between the datasets, we will report both results. If necessary (depending on the outcome of subgroup analyses) we will execute the appropriate approach of combining the aggregated data and the IPD data to perform either: meta-analyses of the aggregated data, meta-analyses of reconstructed IPD or hierarchicalrelated regressions. $^{87}$

\section{Further development of the analysis plan}

We will write a more detailed plan for the statistical analyses in the period from receiving the data to the actual data analyses. In that plan, we will specify how covariates will be modelled (i.e., whether quantitative patient-level characteristics, such as age, is treated as continuous or categorical).

\section{Patient and public involvement}

We are collaborating with three Danish patient and family alliance organisations addressing BPD and mental illness. Representatives from all three organisations have read and commented on the protocol. We are taking this approach to keep the project anchored and in proximity to clinical practice. Here, we indirectly give means to individuals with BPD to influence the research process.

We will similarly invite the members of the patient and family alliance organisations to comment on the IPD reviews before publishing them. We do so to offer a sense of ownership and inclusion in the project.

\section{ETHICS AND DISSEMINATION}

\section{Ethics approval and consent to participate}

Approval by a research ethics committee is not required to conduct these reviews because they involve statistical analyses of anonymous data, that have already been approved by the respective ethical committees that originally assessed the included trials.

\section{Publications}

The three IPD reviews will be published in high-impact factor journals. The results from these reviews will be presented at international conferences as well as in national seminars and conferences.

\section{Author affiliations}

${ }^{1}$ Psychiatric Department, Region Zealand Psychiatry, Psychiatric Research Unit, Slagelse, Denmark

${ }^{2}$ Department of Psychology, University of Southern Denmark Faculty of Health

Sciences, Odense, Denmark

${ }^{3}$ Psychiatric Services, Region Zealand Psychiatry, Roskilde, Denmark ${ }^{4}$ Department of Psychiatry and Psychotherapy, University Medical Centre of the Johannes Gutenberg University Mainz, Mainz, Rheinland-Pfalz, Germany

${ }^{5}$ Psychiatry and Psychotherapy, University Medical Center Mainz, Mainz, Germany

${ }^{6}$ Royal Free and University College Medical School, London, UK

${ }^{7}$ Halliwick Day Unit, St. Ann's Hospital, London, UK
${ }^{8}$ Prof BV Moses Centre for Evidence-Informed Healthcare and Health Policy, Vellore, India

${ }^{9}$ Data and Development Support Unit, Region Zealand, Køge, Denmark

${ }^{10}$ Department of Clinical Psychology, VU University Amsterdam, Amsterdam, The Netherlands

\section{Twitter Eirini Karyotaki @KaryotakiEirini}

Contributors AB, MTK, MSJ, ES, EK, and OJS developed the idea for the protocol. JPR, MSJ, JS-W, KL, AB, MTK, and OJS have drafted the first version of the manuscript. RK, ND, PC, and EK have edited the methodological and statistical section of the manuscript. All authors have critically reviewed and revised the manuscript.

Funding The Psychiatric Research Unit in Region Zealand supported this project with part-time salary for JPR, ES and 0JS during the writing of the manuscript.

Competing interests 0JS: trained in child and adolescent psychoanalytic play therapy, trained in group psychoanalysis and associated with the M-GAB trial. MTK: trained in mentalisation-based and psychodynamic psychotherapy, and conducts research and training in mentalisation-based therapy; has written books on mentalisation-based therapy. JS-W: board-certified behaviour therapist, trained in dialectical behaviour therapy. MSJ: associated with the M-GAB trial, trained in dialectical behaviour therapy and psychodynamic therapy. KL: board-certified cognitive behaviour therapist with a special interest in schema therapy. KL has been involved in trials investigating inpatient dialectical behaviour therapy (Bohus, 2004); and inpatient schema-focused therapy (Reiss, 2014). AB: receives honoraria for training in mentalisation-based treatment for borderline personality disorder. ES: principal investigator of the M-GAB trial, trained in group psychoanalysis.

Patient consent for publication Not required.

Provenance and peer review Not commissioned; externally peer reviewed.

Data availability statement Data sharing not applicable as no datasets generated and/or analysed for this study.

Supplemental material This content has been supplied by the author(s). It has not been vetted by BMJ Publishing Group Limited (BMJ) and may not have been peer-reviewed. Any opinions or recommendations discussed are solely those of the author(s) and are not endorsed by BMJ. BMJ disclaims all liability and responsibility arising from any reliance placed on the content. Where the content includes any translated material, BMJ does not warrant the accuracy and reliability of the translations (including but not limited to local regulations, clinical guidelines, terminology, drug names and drug dosages), and is not responsible for any error and/or omissions arising from translation and adaptation or otherwise.

Open access This is an open access article distributed in accordance with the Creative Commons Attribution Non Commercial (CC BY-NC 4.0) license, which permits others to distribute, remix, adapt, build upon this work non-commercially, and license their derivative works on different terms, provided the original work is properly cited, appropriate credit is given, any changes made indicated, and the use is non-commercial. See: http://creativecommons.org/licenses/by-nc/4.0/.

\section{ORCID iDs}

Ole Jakob Storebø http://orcid.org/0000-0003-0204-7745

Johanne Pereira Ribeiro http://orcid.org/0000-0001-6019-022X

Jutta Stoffers-Winterling http://orcid.org/0000-0001-5286-8133

Eirini Karyotaki http://orcid.org/0000-0002-0071-2599

Pim Cuijpers http://orcid.org/0000-0001-5497-2743

\section{REFERENCES}

1 American Psychiatric Association. Diagnostic and statistical manual of mental disorders (DSM-5). Washington (DC): American Psychiatric Association, 2013

2 Khosravi M. Eating disorders among patients with borderline personality disorder: understanding the prevalence and psychopathology. J Eat Disord 2020;8:ARTN 38.

3 Shah R, Zanarini MC. Comorbidity of borderline personality disorder: current status and future directions. Psychiatr Clin North Am 2018;41:583-93.

4 Tomko RL, Trull TJ, Wood PK, et al. Characteristics of borderline personality disorder in a community sample: comorbidity, treatment utilization, and general functioning. J Pers Disord 2014;28:734-50.

5 Gunderson JG, Herpertz SC, Skodol AE, et al. Borderline personality disorder. Nat Rev Dis Primers 2018;4:1-20. 
6 Bailey RC, Grenyer BFS. Supporting a person with personality disorder: a study of carer burden and well-being. J Pers Disord 2014;28:796-809.

7 Guillén V, Díaz-García A, Mira A, et al. Interventions for family members and carers of patients with borderline personality disorder: a systematic review. Fam Process 2021;60:134-44.

8 Cailhol L, Thalamas C, Garrido C, et al. [Mental health service utilization among borderline personality disorder patients inpatient] Encephale 2015:41:115-22.

9 Hörz S, Zanarini MC, Frankenburg FR, et al. Ten-Year use of mental health services by patients with borderline personality disorder and with other axis II disorders. Psychiatr Serv 2010;61:612-6.

10 Winsper C. Borderline personality disorder: course and outcomes across the lifespan. Curr Opin Psychol 2021;37:94-7.

11 Schneider F, Erhart M, Hewer W. Mortality and medical comorbidity in the severely mentally ill: a German registry study. Deutsches Ärzteblatt International 2019;116:405.

12 Fok ML-Y, Hayes RD, Chang C-K, et al. Life expectancy at birth and all-cause mortality among people with personality disorder. $J$ Psychosom Res 2012;73:104-7.

13 Gunderson JG. Borderline personality disorder. N Engl J Med Overseas Ed 2011;364:2037-42.

14 Gunderson JG, Stout RL, McGlashan TH, et al. Ten-Year course of borderline personality disorder: psychopathology and function from the Collaborative longitudinal personality disorders study. Arch Gen Psychiatry 2011;68:827-37.

15 Winsper C, Bilgin A, Thompson A, et al. The prevalence of personality disorders in the community: a global systematic review and meta-analysis. Br J Psychiatry 2020;216:69-78.

16 Torgersen S. Epidemiology. In: Widiger TA, ed. The Oxford Handbook of personality disorders. New York (NY): Oxford University Press, 2012: 186-205.

17 Hastrup LH, Jennum P, Ibsen R, et al. Societal costs of borderline personality disorders: a matched-controlled nationwide study of patients and spouses. Acta Psychiatr Scand 2019;140:458-67.

18 Goodman M, Patil U, Steffel L, et al. Treatment utilization by gender in patients with borderline personality disorder. J Psychiatr Pract 2010;16:155-63.

19 Stoffers-Winterling J, Storebø OJ, Lieb K. Pharmacotherapy for borderline personality disorder: an update of published, unpublished and ongoing studies. Curr Psychiatry Rep 2020;22:37.

20 Ansell EB, Sanislow CA, McGlashan TH, et al. Psychosocia impairment and treatment utilization by patients with borderline personality disorder, other personality disorders, mood and anxiety disorders, and a healthy comparison group. Compr Psychiatry 2007;48:329-36.

21 Zanarini MC, Frankenburg FR, Reich DB, et al. Treatment rates for patients with borderline personality disorder and other personality disorders: a 16-year study. Psychiatr Serv 2015;66:15-20.

22 Simonsen S, Bateman A, Bohus M, et al. European guidelines for personality disorders: past, present and future. Borderline Personal Disord Emot Dysregul 2019;6:9.

23 Iliakis EA, Sonley AKI, Ilagan GS, et al. Treatment of borderline personality disorder: is supply adequate to meet public health needs? Psychiatr Serv 2019;70:772-81.

24 Bateman AW, Gunderson J, Mulder R. Treatment of personality disorder. Lancet 2015;385:735-43.

25 Kongerslev MT, Chanen AM, Simonsen E. Personality disorder in childhood and adolescence comes of age: a review of the current evidence and prospects for future research. Scand J Child Adolesc Psychiatr Psychol 2015;3:31-48.

26 Storebø OJ, Stoffers-Winterling JM, Völlm BA, et al. Psychological therapies for people with borderline personality disorder. Cochrane Database Syst Rev 2020;5:CD012955.

27 Clarkin JF. An integrated approach to psychotherapy techniques for patients with personality disorder. J Pers Disord 2012;26:43-62.

28 de Groot ER, Verheul R, Trijsburg RW. An integrative perspective on psychotherapeutic treatments for borderline personality disorder. $J$ Pers Disord 2008;22:332-52.

29 Livesley WJ. Moving beyond specialized therapies for borderline personality disorder: the importance of integrated domain-focused treatment. Psychodyn Psychiatry 2012;40:47-74.

30 Weinberg I, Ronningstam E, Goldblatt MJ, et al. Common factors in empirically supported treatments of borderline personality disorder. Curr Psychiatry Rep 2011;13:60-8.

31 Storebø OJ, Stoffers-Winterling JM, Völlm BA. Psychological therapies for people with borderline personality disorder. Cochrane Database Syst Rev 2018:CD012955.

32 Linehan MM. Cognitive-Behavioral treatment of borderline personality disorder. New York (NY): Guilford Press, 1993.
33 Linehan MM. DBT® skills training manual. New York (NY): Guilford Press, 2015.

34 Bateman A, Fonagy P. Psychotherapy for borderline personality disorder: Mentalisation based treatment. Oxford (UK): Oxford University Press, 2004.

35 Bateman A, Fonagy P. Mentalization-Based treatment for borderline personality disorders: a practical guide. Oxford (UK): Oxford University Press, 2006.

36 Bateman A, Fonagy P. Mentalization-Based treatment for personality disorders: a practical guide. Oxford (UK): Oxford University Press, 2016

37 Black DW, Blum N, St John D. Stepps: a practical, evidence-based way to treat borderline personality disorder. Current Psychiatry 2009;8:31-7.

38 Clarkin JF, Yeomans FE, Kernberg OF. Psychotherapy for borderline personality. New York (NY): John Wiley \& Sons, Inc, 1999.

39 Yeomans FE, Clarkin JF, Kernberg OF. Transference-Focused psychotherapy for borderline personality disorder: a clinical guide. Arlington (VA): American Psychiatric Publishing, 2015

40 Chanen AM, McCutcheon L, Kerr IB. HYPE: a cognitive analytic therapy-based prevention and early intervention programme for borderline personality disorder. In: Sharp C TJL, ed. Handbook of borderline personality disorder in children and adolescents. New York (NY): Springer, 2014: 361-83.

41 Ryle A. Cognitive analytic therapy and borderline personality disorder: the model and the method. Chichester (UK): John Wiley \& Sons, Inc, 1997.

42 Arntz A, Van Genderen H. Schema therapy for borderline personality disorder. Chichester (UK): Wiley-Blackwell, 2009.

43 Young JE, Klosko JS, Weishaar ME. Schema Therapy: A Practitioner's Guide. New York (NY): Guilford Press, 2003.

44 Keefe JR, Kim TT, DeRubeis RJ, et al. Treatment selection in borderline personality disorder between dialectical behavior therapy and psychodynamic psychiatric management. Psychol Med 2020:1-9.

45 Barber JP, Solomonov N. Toward a personalized approach to psychotherapy outcome and the study of therapeutic change. World Psychiatry 2019;18:291-2.

46 Navani N, Fisher DJ, Tierney JF, et al. The accuracy of clinical staging of stage I-IIIA non-small cell lung cancer: an analysis based on individual participant data. Chest 2019;155:502-9.

47 Herzog P, Feldmann M, Voderholzer U, et al. Drawing the borderline: predicting treatment outcomes in patients with borderline personality disorder. Behav Res Ther 2020;133:103692.

48 Larun L, Odgaard-Jensen J, Brurberg K. Exercise therapy for chronic fatigue syndrome (individual patient data)(Protocol) 2018.

49 Hiles SA, McDonald VM, Guilhermino M, et al. Does maintenance azithromycin reduce asthma exacerbations? an individual participant data meta-analysis. Eur Respir J 2019;54:1901381.

50 Panagioti M, Bower P, Kontopantelis E, et al. Association between chronic physical conditions and the effectiveness of collaborative care for depression: an individual participant data meta-analysis. JAMA Psychiatry 2016;73:978-89.

51 Tucker KL, Sheppard JP, Stevens R, et al. Self-monitoring of blood pressure in hypertension: a systematic review and individual patient data meta-analysis. PLoS Med 2017;14:e1002389.

52 Boschloo L, Bekhuis E, Weitz ES, et al. The symptom-specific efficacy of antidepressant medication vs. cognitive behavioral therapy in the treatment of depression: results from an individual patient data meta-analysis. World Psychiatry 2019;18:183-91.

53 Cuijpers P, Weitz E, Twisk J, et al. Gender as predictor and moderator of outcome in cognitive behavior therapy and pharmacotherapy for adult depression: an "individual patient data" meta-analysis. Depress Anxiety 2014;31:941-51.

54 Driessen E, Dekker JJM, Peen J, et al. The efficacy of adding short-term psychodynamic psychotherapy to antidepressants in the treatment of depression: a systematic review and meta-analysis of individual participant data. Clin Psychol Rev 2020;80:101886.

55 Furukawa TA, Efthimiou O, Weitz ES, et al. Cognitive-Behavioral analysis system of psychotherapy, drug, or their combination for persistent depressive disorder: Personalizing the treatment choice using individual participant data network metaregression. Psychother Psychosom 2018;87:140-53.

56 Karyotaki E, Ebert DD, Donkin L, et al. Do guided Internet-based interventions result in clinically relevant changes for patients with depression? an individual participant data meta-analysis. Clin Psychol Rev 2018;63:80-92.

57 Karyotaki E, Kemmeren L, Riper H, et al. Is self-guided internetbased cognitive behavioural therapy (iCBT) harmful? An individual participant data meta-analysis. Psychol Med 2018;48:2456-66. 
58 Riley RD, Lambert PC, Abo-Zaid G. Meta-analysis of individual participant data: rationale, conduct, and reporting. BMJ 2010;340:c221.

59 Higgins JPT, Thomas J, Chandler J. Cochrane Handbook for systematic reviews of interventions. Chichester (UK: John Wiley \& Sons, 2019.

60 Stewart LA, Clarke M, Rovers M, et al. Preferred reporting items for systematic review and meta-analyses of individual participant data: the PRISMA-IPD statement. JAMA 2015;313:1657-65.

61 Sterne JAC, Savović J, Page MJ, et al. Rob 2: a revised tool for assessing risk of bias in randomised trials. BMJ 2019;366:14898.

62 Pufulete M, Higgins JP, Rogers CA, et al. Protocol for a systematic review and individual participant data meta-analysis of B-type natriuretic peptide-guided therapy for heart failure. Syst Rev 2014;3:41.

63 Zanarini MC, Vujanovic AA, Parachini EA, et al. Zanarini rating scale for borderline personality disorder (ZAN-BPD): a continuous measure of DSM-IV borderline psychopathology. J Pers Disord 2003;17:233-42.

64 Arntz A, van den Hoorn M, Cornelis J, et al. Reliability and validity of the borderline personality disorder severity index. J Pers Disord 2003;17:45-59.

65 Perez V, Barrachina J, Soler J, et al. The clinical global impression scale for borderline personality disorder patients (CGI-BPD): a scale sensible to detect changes. Actas Esp Psiquiatr 2007;35:229-35.

66 Horowitz LM, Rosenberg SE, Baer BA, et al. Inventory of interpersonal problems: psychometric properties and clinical applications. J Consult Clin Psychol 1988;56:885-92.

67 Ritsner M, Kurs R, Gibel A, et al. Validity of an abbreviated quality of life enjoyment and satisfaction questionnaire (Q-LES-Q-18) for schizophrenia, schizoaffective, and mood disorder patients. Qual Life Res 2005;14:1693-703.

68 Herdman M, Gudex C, Lloyd A, et al. Development and preliminary testing of the new five-level version of EQ-5D (EQ-5D-5L). Qual Life Res 2011;20:1727-36.

69 Endicott J, Spitzer RL, Fleiss JL, et al. The global assessment scale. A procedure for measuring overall severity of psychiatric disturbance. Arch Gen Psychiatry 1976;33:766-71.

70 American Psychiatric Association. Diagnostic and statistical manual of mental disorders (DSM-III-R. Washington (DC: American Psychiatric Association, 1987.

71 Tyrer P, Nur U, Crawford M, et al. The social functioning questionnaire: a rapid and robust measure of perceived functioning. Int J Soc Psychiatry 2005;51:265-75.

72 Gratz KL. Measurement of deliberate self-harm: preliminary data on the deliberate self-harm inventory. J Psychopathol Behav Assess 2001;23:253-63.
73 Gutierrez PM, Osman A, Barrios FX, et al. Development and initial validation of the self-harm behavior questionnaire. J Pers Assess 2001;77:475-90

74 Osman A, Bagge CL, Gutierrez PM, et al. The suicidal behaviors Questionnaire-Revised (SBQ-R): validation with clinical and nonclinical samples. Assessment 2001;8:443-54.

75 Beck AT, Kovacs M, Weissman A. Assessment of suicidal intention: the scale for suicide ideation. J Consult Clin Psychol 1979;47:343-52.

76 Levine J, Schooler NR. SAFTEE: a technique for the systematic assessment of side effects in clinical trials. Psychopharmacol Bull 1986;22:343-81.

77 International Council for Harmonisation Expert Working Group. International Conference on harmonisation of technical requirements for registration of pharmaceuticals for human use. $\mathrm{ICH}$ harmonised tripartite guideline. Guideline for good clinical practice E6(R1) 2011 wwwichorg/fileadmin/Public_Web_Site/ICH_Products/Guidelines/ Efficacy/E6/E6 R1 Guidelinepdf

78 Breedvelt JJF, Warren FC, Brouwer ME, et al. Individual participant data (IPD) meta-analysis of psychological relapse prevention interventions versus control for patients in remission from depression: a protocol. BMJ Open 2020;10:e034158.

79 Jakobsen JC, Wetterslev J, Winkel P, et al. Thresholds for statistical and clinical significance in systematic reviews with meta-analytic methods. BMC Med Res Methodol 2014;14:120.

80 Burke DL, Ensor J, Riley RD. Meta-analysis using individual participant data: one-stage and two-stage approaches, and why they may differ. Stat Med 2017;36:855-75

81 Belias M, Rovers MM, Reitsma JB, et al. Statistical approaches to identify subgroups in meta-analysis of individual participant data: a simulation study. BMC Med Res Methodol 2019;19:183.

82 Janssen KJM, Donders ART, Harrell FE, et al. Missing covariate data in medical research: to impute is better than to ignore. $J$ Clin Epidemiol 2010;63:721-7.

83 Little RJ, D'Agostino R, Cohen ML, et al. The prevention and treatment of missing data in clinical trials. N Engl J Med 2012;367:1355-60.

84 Steyerberg EW, Nieboer D, Debray TPA, et al. Assessment of heterogeneity in an individual participant data meta-analysis of prediction models: an overview and illustration. Stat Med 2019;38:4290-309.

85 StataCorp L. Stata statistical software: release 16. College Station, TX: StataCorp, 2019

86 de Zoete A, de Boer MR, van Tulder MW, et al. Rational and design of an individual participant data meta-analysis of spinal manipulative therapy for chronic low back pain-a protocol. Syst Rev 2017;6:21.

87 Debray TPA, Moons KGM, van Valkenhoef G, et al. Get real in individual participant data (IPD) meta-analysis: a review of the methodology. Res Synth Methods 2015;6:293-309. 